\title{
LA TRANSVERSALIDAD DE LA
} EDUCACIÓN AMBIENTAL EN DOS INSTITUCIONES EDUCATIVAS DEL DEPARTAMENTO DE NARIÑO, COLOMBIA

\section{THE TRANSVERSALITY OF}

\section{ENVIRONMENTAL EDUCATION IN TWO EDUCATIONAL INSTITUTIONS OF THE DEPARTMENT OF NARIÑO, COLOMBIA}

\author{
Rivas-Escobar, Hernan Modesto ${ }^{1}$ \\ Luna-Cabrera, Gloria Cristina ${ }^{2}$ \\ Moreno-Molina, Angela Andrea ${ }^{3}$ \\ Universidad de Nariño
}

\footnotetext{
1 HERNAN MODESTO RIVAS ESCOBAR: 00000002-3978-4952

Docente Departamento de Estudios Pedagógicos de la Facultad de Educación, de la Universidad de Nariño, Investigador grupo Pifil. Hernan.rivas@udenar.edu.co 2 GLORIA CRISTINA LUNA CABRERA: 00000001-5433-1970

Docente Departamento de Recursos Naturales y Sistemas Agroforestales de la Facultad de Ciencias Agrícolas de la Universidad de Nariño, directora grupo de investigación PIFIL.cristinalunac@udenar.edu.co

3 ANGELA ANDREA MOLINA MORENO: 00000003-3192-3206

Docente Departamento de Recursos Naturales y Sistemas Agroforestales de la Facultad de Ciencias Agrícolas de la Universidad de Nariño, investigadora grupo PIFIL. Angela. molina@udenar.edu.co
}

\section{RESUMEN}

La transversalidad es la ruta que hemos recorrido para avanzar hacia la construcción de una realidad compleja donde se integra lo disciplinar del tema ambiental con las construcciones académicas que unen la experiencia y la realidad al currículo. El trabajo se desarrolló en dos instituciones educativas públicas del departamento de Nariño, Colombia, una ubicada en el sector rural y otra en un sector urbano. Las dos Instituciones han participado del proyecto de transversalidad de la educación ambiental apoyado por la Vicerrectoría de Investigaciones e Interacción Social de la Universidad de Nariño. El objetivo de la investigación fue determinar y analizar la forma en la que se está transversalizando la dimensión ambiental en 
el currículo escolar a través de los proyectos ambientales escolares PRAE, conocer las percepciones de los docentes frente a conceptos relevantes en el proceso y cómo esa visión afecta la operacionalización y ejecución del proyecto de transversalidad. Se empleo una metodología dentro del paradigma cualitativo enmarcada en la Investigación Acción Participativa, empleando conversatorios y encuestas con preguntas abiertas que se procesaron con la ayuda del software Atlas ti. Se elaboraron unas categorías que permitieron determinar las construcciones conceptuales de los docentes y las habilidades institucionales para transversalizar la dimensión ambiental en sus currículos escolares.

\section{PALABRAS CLAVE:}

educación ambiental, transversalidad, currículo, ambiente, PRAE

\section{ABSTRACT}

Transversality is the route that we have traveled to advance towards the construction of a complex reality where the disciplinary of the environmental issue is integrated with the academic constructions that unite experience and reality to the curriculum. The work was developed in two public educational institutions in the department of Nariño, Colombia, one located in the rural sector and the other in an urban sector. The two Institutions have participated in the project of transversality of environmental education supported by the Office of the Vice Rectory for Research and Social Interaction of the University of Nariño. The objective of the research was to determine and analyze the way in which the environmental dimension is being mainstreamed in the school curriculum through the PRAE school environmental projects, to know the perceptions of teachers regarding relevant concepts in the process and how that vision it affects the operationalization and execution of the transversality project. A methodology was used within the qualitative paradigm framed in Participatory Action Research, using conversations and surveys with open questions that were processed with the help of the Atlas ti software. Some categories were elaborated that allowed to determine the conceptual constructions of the teachers and the institutional abilities to mainstream the environmental dimension in their school curricula.

\section{KEY WORDS:}

environmental education, mainstreamed, curriculum, environmental, PRAE.

\section{RESUMO}

A transversalidade é o caminho que percorremos para avançar rumo à construção de uma realidade complexa onde a disciplina da questão ambiental se integra às construções acadêmicas que unem experiência e realidade ao currículo. O trabalho foi desenvolvido em duas instituições públicas de ensino do departamento de Nariño, Colômbia, uma localizada no setor rural e a outra no setor urbano. As duas Instituições participaram do projeto de transversalidade da educação ambiental apoiado pela Vice-Presidência de Pesquisa e Interação Social da Universidade de Nariño. O objetivo da pesquisa foi determinar e analisar a forma como a dimensão ambiental está sendo inserida no currículo escolar por meio dos projetos ambientais escolares do PRAE, para conhecer as percepções dos professores sobre conceitos relevantes no processo e como essa visão afeta os operacionalização e execução do projeto de transversalidade. Foi utilizada uma metodologia dentro do paradigma qualitativo enquadrado na Pesquisa-Ação Participativa, utilizando conversas e questionários com questões abertas que foram processadas com o auxílio do software Atlas ti. Elaboraram-se algumas categorias que permitiram determinar as construções conceituais dos professores e as habilidades institucionais para integrar a dimensão ambiental em seus currículos escolares. 


\section{PALAVRAS-CHAVE:}

educação ambiental, integração, currículo, meio ambiente, PRAE

\section{INTRODUCCIÓN}

La concepción de ambiente es precedida por la concepción de naturaleza y arrastra una connotación desde la modernidad. Para indagar acerca de la concepción de educación ambiental y de la transversalidad de esta en los diseños curriculares, debemos explorar bajo qué entendido de ambiente vamos a recorrer ese camino.

Una de las características de la modernidad es la renuncia del hombre a entenderse a sí mismo como parte de la naturaleza, en el pensamiento clásico anterior a la modernidad la filosofía política, incluida la griega, giraba en torno a una idea definitiva de la naturaleza, (Innerarity, 1987), manifiesta que es el lugar metafísico donde el hombre expresaba su deseo de estar amparado y protegido por ella y por su accionar como ente. La consideración de la naturaleza como ser es profunda y ese en particular es un hecho de quiebre epistemológico radical de la modernidad frente al valor de uso que esta le da a lo natural.

En la visión de la modernidad el ejercicio epistemológico de conocer se asimila a la dominación de un objeto externo, extraño al mismo hombre y la condición de la naturaleza que posteriormente evoluciona hacia el concepto de ambiente, pero igualmente alejado de un valor intrínseco que no va más allá del valor que pueda darle el ser humano, allana el camino a la construcción de una ideología de la dominación del hombre sobre el mundo, (AngelMaya, 2014).

El hecho de que el hombre le quite al ambiente su valor innato es la declaración de renuncia a comprenderse como parte de él, esta condición es el sustento de la duda metódica cartesiana, que incluso se puede evidenciar en Hobbes y Freud.

Vale la pena hacer diferencia, más que semántica en lo que es la modernidad como proyecto humano y la época moderna. El proyecto moderno laico como alternativa al oscurantismo conservador y reaccionario es legítimo, pero no es legítimo, al decir de Rafael Alvira, (2007), lo que conocemos desde hace tres siglos como modernidad, ni tampoco es legítimo la interpretación que esa modernidad hace de los últimos 500 años, época conocida como modernidad.

La educación, incluida la ambiental, es un instrumento del establecimiento para perpetuar el statu quo, que permite la reproducción de las estructuras de poder, dominación y alienación ideológica; sin embargo, también puede ser el escenario para la construcción de ideas de transformación y cambio. Para el análisis de la educación ambiental, coincidimos con BarreroGarcia (2019), en que deberíamos considerar el hecho de que el proyecto moderno, sustento epistemológico de la forma como configuramos o creemos configurar nuestros proyectos de vida individuales y colectivos y de la forma que como especie nos relacionamos con las otras especies del ecosistema planetario con las que hemos coevolucionado sabiamente, hasta ahora, por millones de años; es precisamente el origen de la crisis ambiental. En este orden de ideas ¿cómo podemos confiar en que la ciencia y la tecnología producto de esa construcción cultural nos van a ayudar a construir el arca si ellos provocaron el diluvio?

Distinguimos la transversalidad en la educación ambiental en dos perspectivas, una es la transversalidad interdisciplinar y otra la transversalidad transdisciplinar. Según Gutiérrez (1995), la transversalidad interdisciplinar se aborda como un campo del conocimiento que tiene una identidad propia a la que las otras áreas del conocimiento entendidas como 
asignaturas escolares le hacen sus respectivos aportes, aunque esta mantiene su condición de asignatura dentro del plan de estudios y desarrolla contenidos específicos como cualquier otra materia del pensum escolar. Los contenidos que puede desarrollar son por ejemplo, el cambio climático, la contaminación, los residuos sólidos, la biodiversidad, las energías verdes, entre otros.

En éste sentido, la transversalidad transdisciplinar deja de tener la característica de asignatura específica para transformarse en una materia integrada que transita por todas las áreas del conocimiento escolar institucionalizado. Según Gutiérrez y Pozo, (2006) se pueden desarrollar dos formas para esta perspectiva transdisciplinar: en primer lugar está la transversalidad transdisciplinar típica donde dentro de cada unidad didáctica de los contenidos de las diversas asignaturas, aparecen de forma sistemática aspectos específicos y contenidos propios de los diferentes ejes transversales. Sobre esos mismos núcleos temáticos se construyen y desarrollan las propuestas curriculares de la educación ambiental, en función del tema que haya que tratar (Luna-Cabrera, et.al 2020).

De otra parte, la transversalidad entendida como principio didáctico de conexión disciplinar con los estándares o derechos básicos de aprendizaje de cada asignatura en particular. Un ejemplo es el ejercicio hecho por LunaCabrera, et.al (2020), donde la filosofía puede aplicarse a la construcción de soluciones de una problemática relacionada con el recurso hídrico, en este caso el estándar es "identifico los principales planteamientos de las diferentes reflexiones en torno al origen del cosmos en el principio generador", desde este escenario se plantea para la construcción de los aprendizajes la cosmogonía del universo partiendo de un fundamento común: el agua.
Desde la perspectiva múltiple del constructivismo social, la teoría crítica y la complejidad, la educación ambiental persigue la aprehensión y construcción de competencias, conceptos, habilidades y valores que expliquen las relaciones ecosistémicas, los ciclos biogeoquímicos, los flujos de energía, la biodiversidad y las relaciones sociales y culturales, desde escenarios donde la interdisciplinariedad actúa transversalmente. Esto nos lleva a un nuevo escenario didáctico que metodológicamente es un camino por construir, donde deben confluir las diferentes disciplinas y sus respectivos actuares pedagógicos que se desarrollan bajo estrategias metacognitivas porque se deben habilitar ciudadanos que puedan dar soluciones incluso a problemas que aún no se han presentado. (Laso-Salvador, 2018).

Otro enfoque acerca de cómo podemos ambientalizar el currículo lo plantea la Unesco en el libro de Consulta "Educación para el desarrollo sostenible" (2012), en un relacionamiento entre los conceptos de sociedad, economía y sostenibilidad ambiental. Un reto para la transversalización y ambientalización de las estructuras curriculares son los problemas ambientales y la sostenibilidad, por ejemplo, como los servicios ecosistémicos de captura de carbono, conservación de la biodiversidad, regulación hídrica o calidad paisajística se ven afectados por actividades productivas particulares en entornos específicos. En este ejemplo se puede desarrollar los temas productivos de medio y alto impacto como la minería u otras actividades extractivas en zonas de importancia ambiental. (UNESCO, 2012).

Solano (2006) citando a Colom y Sureda (1989), en su trabajo acerca de la historia de la educación ambiental en Costa Rica, menciona que en los inicios del siglo XXI se espera que la educación ambiental sea un componente más del currículo $y$ no un eje transversal, cuando en nuestra opinión lo que ha sucedido es básicamente todo 
lo contrario. La aspiración debe ser integrar la dimensión ambiental al currículo, Luna, Molina, Leonel y Rivas (2020):

"En el currículo podemos expresar entonces la posición disciplinar o la transdisciplinar, $\mathrm{O}$ todos los puntos intermedios entre estas dos formas de acercarse al conocimiento. Lo que estamos proponiendo, por considerarlo más cercano a la realidad del mundo de la vida, es la complejización del saber cómo lo proponen Leff (2006), Morín (2000), Sabogal (2009), entre otros".

El departamento de Nariño, Colombia, es una región biodiversa y pluricultural, donde convergen múltiples sistemas naturales, culturales y sociales, con una complejidad amplia en el abordaje de los procesos de gestión ambiental con comunidades, instituciones y diferentes actores (Osorio 2015). Considerando esto, es oportuno entender, que el objeto de la educación ambiental no se encuentraúnicamente determinado a analizar los elementos que determinan el daño al ecosistema planetario y su impacto sobre la especie humana; sino también, alcanza las opciones de solución desde una visión social, política, económica, ética, jurídica y cultural (Rodríguez and García 2005).

Convertir la dimensión ambiental en un sector o en un elemento aislado del currículo es regresivo, partimos de la afirmación de que toda la educación debe ser ambiental, y esto se traduce en una ambientalización total de los contenidos curriculares, un ejemplo de esto tomado un ejercicio con el recurso hídrico y los residuos sólidos en una institución educativa del municipio de Chachagüí en el departamento de Nariño Colombia (Luna-Cabrera, et al 2020).

En este sentido el presente estudio hace parte de una investigación mayor denominada "Procesos de transversalidad curricular de la dimensión ambiental en tres instituciones educativas del municipio de Pasto, departamento de Nariño", adelantada en el grupo de investigación PIFIL, de la Universidad de Nariño y que aspira poder comprender los aspectos institucionales y pedagógicos que promueven o dificultan la transversalidad de la dimensión ambiental en el currículo. Identificar aspectos importantes para plantear la transversalidad de la dimensión ambiental en el currículo, analizando el estado actual de los proyectos ambientales escolares PRAE y realizando el diagnóstico ambiental en cada institución educativa con el fin de determinar alternativas ambientales pedagógicas para la transversalidad curricular pertinente para cada una de las instituciones educativas y formular indicadores de gestión, seguimiento y evaluación que permitan un proceso de mejoramiento continuo.

De ésta manera se pretende contribuir con una orientación para el proceso de transversalización y propuestas pedagógicodidácticas que contemplen el diálogo de saberes y coherencia entre actitudes y comportamientos ambientales dirigidos a la comunidad educativa, con el propósito de reconocer la estrategia los proyectos ambientales escolares PRAE en el desarrollo de la educación ambiental hacia la trasformación de actitudes ambientales.

\section{MATERIALES Y MÉTODOS}

El estudio se adelantó con la participación de docentes de la Institución Educativa Municipal Chachagui- IEMCh y la Institución Educativa Municipal Morasurco- IEMM del departamento de Nariño en Colombia, quienes frente a la actual afectación ambiental pretenden orientar la formación de los estudiantes desde el análisis de su realidad mediante los proyectos ambientales escolares.

La metodología de la investigación está dentro del paradigma cualitativo enmarcada en la Investigación Acción Participativa, en la medida que se espera con el acompañamiento 
horizontal a los actores educativos transformar una realidad de los entornos escolares, se utilizarón instrumentos y herramientas de captura y análisis de información de tipo mixto. Para el procesamiento y análisis de la información se construyeron las siguientes categorías: ambiente, educación ambiental, currículo, transversalidad. La información se obtuvo mediante la observación directa en el acompañamiento a los diagnósticos participativos para la resignificación de los PRAE y a los talleres de construcción de alternativas a los problemas ambientales identificados en los entornos escolares y la posterior articulación de esa problemática a los planes de estudio y su conexión con los estándares básicos por competencias.

La información de los docentes participantes de las Instituciones educativas de Chachagui y Morasurco se complementó con las encuestas y entrevistas que se procesaron con el software Atlas ti. Para la revisión de la literatura también se utilizó el Atlas ti con el fin de construir las unidades hermenéuticas y las redes semánticas que contrastan y validan la práctica con la teoría pedagógica.

Según Luna-Cabrera et al (2020), los Proyectos Educativos Ambientales Escolares (PRAE), como herramienta pedagógica permiten el análisis de la problemática ambiental escolar, hacia la búsqueda de soluciones acordes a las realidades de cada institución, dentro de los contextos natural, sociocultural, político y económico, puesto que este:

"Brinda una oportunidad de reorientar modelos tradicionales, maximizar la participación y empoderamiento de miembros de la comunidad educativa, instituciones del sector y organizaciones sociales a través de la integración de conocimientos y experiencias de diferentes dinámicas curriculares de manera transversal en torno a un objetivo".

El problema es el reduccionismo y el asignaturismo de los currículos escolares, direccionados desde la orientación de la política pública educativa basada en unos estándares básicos por competencias y recientemente en los derechos básicos de aprendizaje que limita y circunscribe lo que se puede enseñar a una estructura rígida presionada, además, por la posibilidad de que las instituciones educativas puedan obtener mejores resultados en las pruebas estandarizadas. Los discursos pedagógicos inscritos en los documentos oficiales como los Proyectos Educativos Institucionales teorizan acerca de modelos pedagógicos eclécticos donde la libertad de cátedra, la pertinencia y la significancia junto al protagonismo del estudiante se ponderan como principios rectores de su quehacer, pero en la práctica no se pueden abandonar las viejas tácticas y estrategias del pensar positivo, reduccionista que promueve y mantiene vigente aún hoy en día el conductismo.

En ese sentido, Colombia es un país contradictorio, como le expresa Arce-Tovar, (2020), a pesar de ser considerados como uno de los países de la región con una normatividad liberal y llena de garantías de derechos en los temas ambientales, partiendo desde la misma constitución política, considerada la constitución verde de América y de tener una reglamentación extensa en los temas ambientales que se aterrizan en la educación ambiental a través de la ley 115 de 1994, el decreto 1743 de 1994, la ley 1549 del 2012, el decreto 1076 del 2015, entre muchos otros; la práctica o la realidad que nos permite la lectura de la vida diaria es muy diferente. Existe una ruptura entre las intensiones y los discursos oficiales y las prácticas sociales que se vivencian en las instituciones educativas y sus áreas de influencia. Los PRAE, se han venido formulando y aplicando durante 27 años 
y su estructura no ha cambiado a pesar de estar viviendo un escenario ambiental muy diferente al ahora lejano 1994.

Parte del problema es la indeterminación conceptual de los protagonistas del proceso educativo, especialmente de los docentes que tienen la oportunidad de comunicar, a veces equivocadamente, un mensaje disciplinar cuando en realidad se piensa en ejercicios complejos y transdisciplinares. Esto se supera considerando y explorando los referentes conceptuales y contrastando con los pares y con las construcciones teóricas para después volverlo a cotejar con los mismos actores y así reconfigurar la concepción conceptual y también reconstruir la teoría desde la propia experiencia.

El problema identificado está relacionado con la diversidad de opiniones, concepciones y percepciones acerca de los conceptos fundamentales de transversalidad, educación y ambiente. Dependiendo de lo que los actores educativos entiendan por cada uno de los conceptos mencionados se podrá desarrollar un modelo o enfoque de educación ambiental y se podrá también estructurar una estrategia para transversalizar la educación ambiental en los escenarios curriculares de las instituciones educativas.

Al indagar acerca de las concepciones de transversalidad y educación ambiental se propicia una reflexión acerca del papel de los proyectos ambientales escolares (PRAE), en la incorporación de la dimensión ambiental en el quehacer educativo de las instituciones de enseñanza de los niveles de educación básica primaria, secundaria y media.

Aunque prevalece una visión antropocentrista del ambiente heredada de la formación disciplinar de los participantes inmersos en un sistema academicista y en el marco de la educación por competencias donde los contenidos están determinados por los estándares básicos, recientemente apoyados por los llamados Derechos Básicos de Aprendizaje promulgados e impuestos por el Ministerio de Educación Nacional (MEN, 2016), se vislumbran concepciones más cercanas al biocentrismo, con una visión más holística y compleja del ambiente que permite integrar y abordar la dimensión ambiental desde las diferentes disciplinas a través de la implementación de proyectos que den solución a problemas específicos de la comunidad educativa. (Pita-Morales, 2016)

Otro elemento a considerar en este análisis es la forma en la que el establecimiento a pretendido ligar el concepto de desarrollo sostenible a todas las iniciativas públicas, privadas $\mathrm{y}$ ciudadanas que tienen preocupación por el asunto ambiental, en este sentido consideramos que hay un problema ético fundamental ligado al concepto del desarrollo sostenible o sustentable, y este radica en que su propuesta intrínseca está ligada al origen primigenio y semántico del término y del concepto, en el que propone la sostenibilidad del desarrollo como un fin intergeneracional de la humanidad, ubicándolo como un valor supremo de la sociedad al que deben plegarse otros valores como el respeto, las solidaridad, la cooperación, la corresponsabilidad. El subterfugio ético está en que no se exceda la capacidad de carga del ambiente mientras se satisface el voraz apetito consumista de la sociedad moderna actual y futura, (Sauve, 1999).

Nuestros docentes deben tener muy claro lo que significa la educación para el desarrollo sostenible y porque la educación ambiental es rebelde y alternativa desde su visión de territorio, su concepción de bien vivir, y sobre la construcción de una ética para la generación de un nuevo ciudadano planetario con respeto y valoración de su identidad en un mundo diverso. Hacemos esta consideración debido al sentido que los organismos nacionales e internacionales le están tratando de dar a la educación ambiental 
propiciando un giro a lo que llaman educación para el desarrollo sostenible.

\section{RESULTADOS Y DISCUSIÓN}

Currículo, competencias y estándares básicos por competencias

Los docentes de las instituciones educativas que participaron de esta investigación tienen formación universitaria y muchos de ellos poseen formación de posgrado, y se desempeñan en diferentes áreas del conocimiento con experiencia en el sector educativo de más de 12 años; quienes fueron consultados sobre sus conocimientos y percepciones acerca del currículo, los estándares y las competencias, en el marco del desarrollo del proyecto de transversalización de la dimensión ambiental que inicialmente diagnosticó participativamente la problemática ambiental sustento de los proyectos ambientales escolares (PRAE), y que posteriormente las soluciones propuestas fueron incluidas en los planes de estudio de los diferentes grados de los niveles de educación básica y media.

Partimos del hecho que el sistema educativo Colombiano está basado en un modelo de competencias, lafrancesco (2004), donde los estándares básicos por competencias y más recientemente los derechos básicos de aprendizaje (DBA), entendidos según el MEN (2016), como "un conjunto de aprendizajes estructurantes que han de aprender los estudiantes en cada uno de los grados de educación escolar, desde transición hasta once", delimitan la construcción curricular en los niveles de educación básica y media. Los docentes de las instituciones educativas de Chachagui y Morasurco, tienen nivel educativo universitario y la gran mayoría han adelantado estudios de posgrado a nivel de especialización y maestría, como se puede observar en la figura No. 1, esto es relevante en el momento de analizar las respuestas a las preguntas conceptuales de competencias, estándares básicos por competencias y currículo.

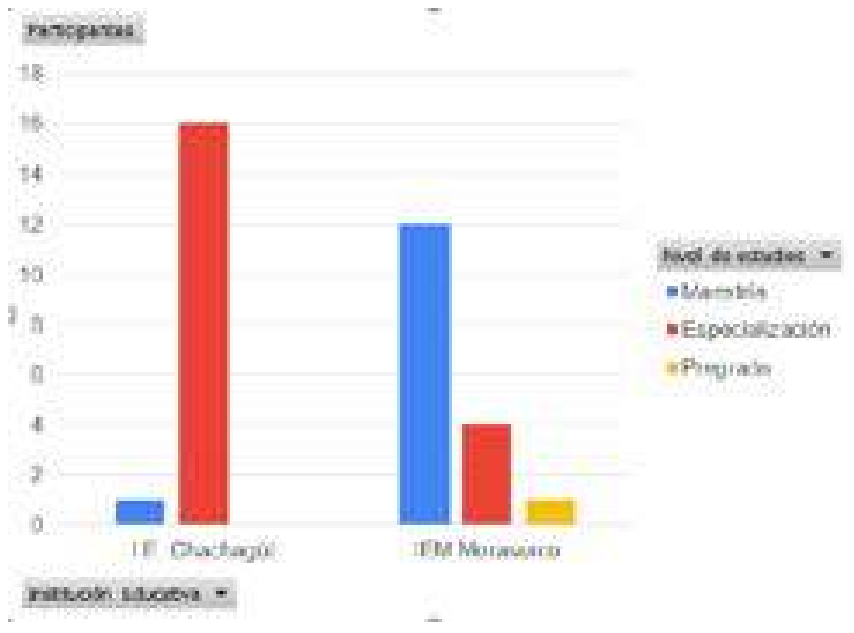

Figura No. 1. Nivel académico de los participantes de la investigación. Fuente: este estudio

El análisis en conjunto de las categorías de currículo, estándares y competencias, permitieron crear una supra categoría que al analizarse bajo un diagrama de coocurrencias Sankey, Figura No. 2, nos permite identificar coincidencias en la visión educativa centrada en el estudiante propio de la escuela activa que ha contribuido al giro epistémico del quehacer pedagógico preponderante en la escuela tradicional, Aldana-Zavala (2021). Igualmente se destaca en la visión de los docentes los saberes y las habilidades como elementos donde se conjuga la experiencia curricular con los estándares y las competencias para el logro de lo que ellos llaman una formación integral. Se puede construir la analogía de los saberes con la competencia general del saber conocer y la categoría habilidades con la competencia genérica del saber hacer y la concepción de la formación integral con el saber ser.

Las anteriores consideraciones se reflejan en la afirmación de uno de los docentes participantes de esta investigación al decir: 
5:10 I 20, "El currículo es el conjunto de procesos que orientan la formación de los estudiantes en una institución. Entre ellos se encuentra el plan de estudios, pero no se limita sólo a los contenidos académicos de este, sino que se acompaña del conocimiento del contexto donde se trabaja. Por lo tanto, las características del contexto nutren el quehacer educativo $y$ promueve un currículo acorde con los aspectos culturales, sociales, económicos y de aprendizaje del lugar en donde se encuentra una institución. El currículo permea a todos los actores de la educación: docentes, estudiantes, padres de familia para alcanzar un proyecto educativo acorde con las necesidades de la comunidad" in Case 5

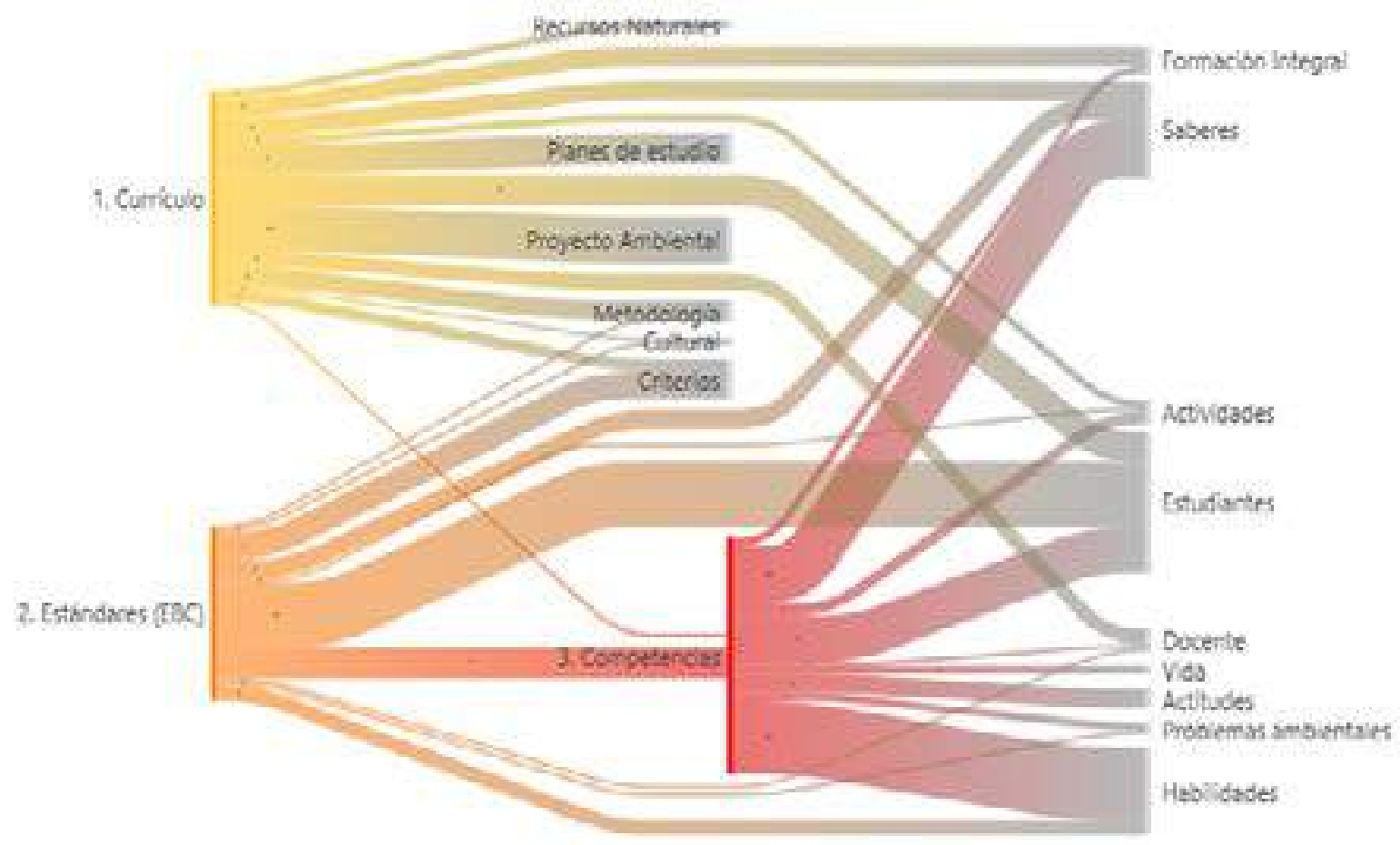

Figura No 2. Relación entre las categorías de currículo, estándares y competencias.

Fuente: este estudio

\section{EDUCACIÓN AMBIENTAL}

Las construcciones conceptuales alrededor de la educación ambiental nos ayudan a articular la idea de los docentes con la macro categoría de Currículo, competencias y estándares básicos por competencias y la categoría de transversalidad de la dimensión ambiental en el currículo. Utilizando un análisis de co- ocurrencia de las respuestas a la encuesta realizada a los docentes de las Instituciones Educativas Morasurco y Chachagüí, mediante la utilización del Atlas ti, podemos establecer que los mayores niveles de relacionamiento frente a la pregunta abierta de ¿Qué es para usted la educación ambiental?, están en las nociones de conciencia, medio ambiente, saberes, recursos 
naturales y vida, tal como puede observarse en el diagrama de Sankey de la figura No 3.

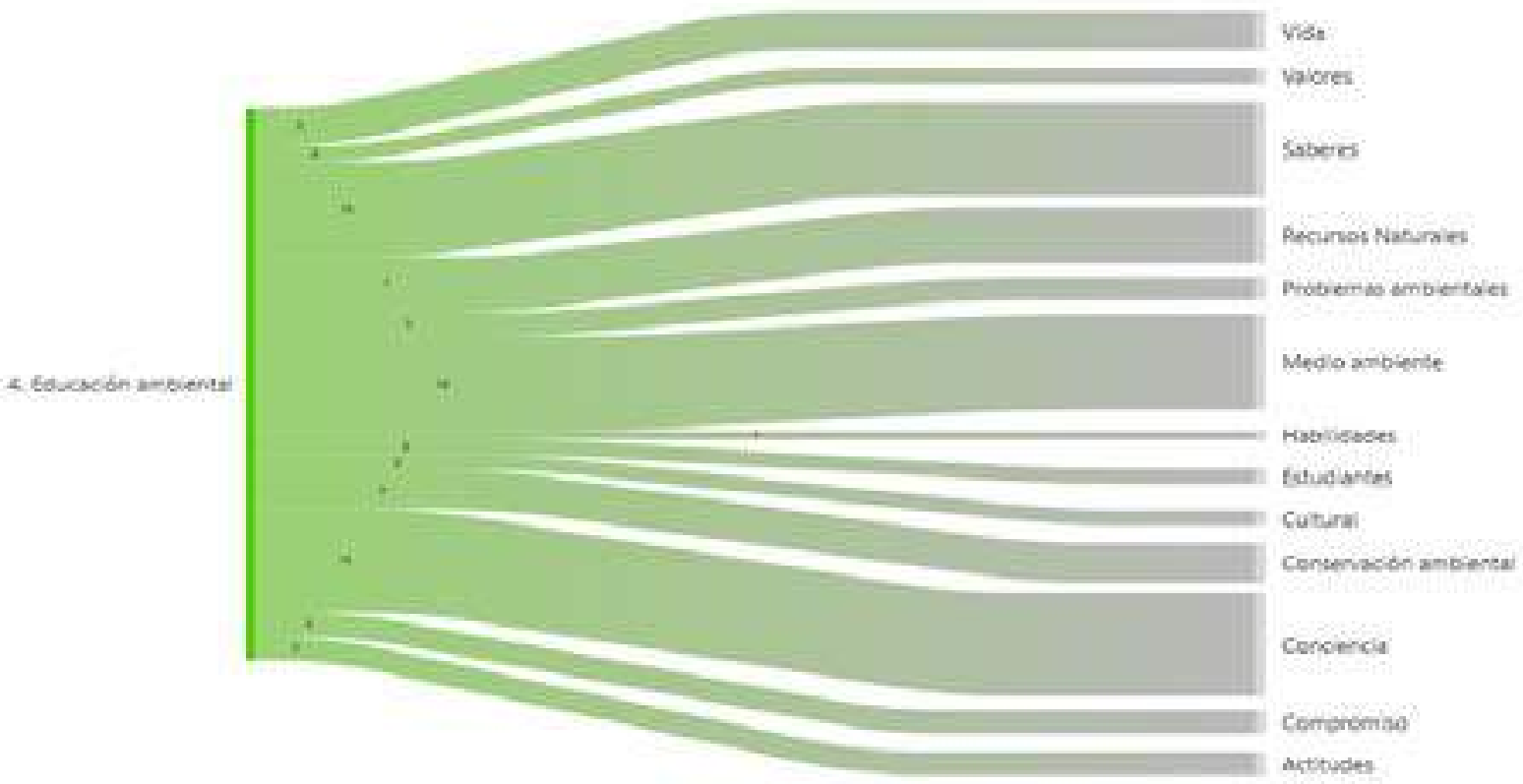

Figura No. 3. Consideraciones de los docentes de la frente a la educación ambiental.

Fuente: este estudio

Las relaciones que los docentes participantes de este estudio hacen de la educación ambiental con la conciencia, con los saberes, con la vida, con el ambiente, con las actitudes y compromisos son una demostración de que el espíritu emergente de la complejidad está captando el interés del sector educativo.

En la construcción conceptual y en la búsqueda de los referentes teóricos y metodológicos de la educación ambiental, existen muchas variantes y múltiples identidades, debido a la variedad de objetivos y propósitos que se encuentran en las variadas prácticas de educación ambiental presentes en la sociedad y al interior de las instituciones educativas. Esto nos lleva en primer lugar, a reconocer que existe una gran variedad de prácticas educativas, y que consecuentemente, existen variados modelos y posturas epistemológicas que los sustentan, algunos de manera implícita y otros de forma explícita. Algunos actores de las prácticas de educación ambiental son conscientes del respaldo teórico de sus acciones, otros actúan de forma espontánea y reactiva frente a situaciones específicas y de acuerdo con su pensar y sentir, sin deliberar el trasfondo epistemológico de sus actos (Gutierrez \& Pozo, 2006).

Buscar en la multiplicidad de opciones y tendencias actuales de la educación ambiental, sus objetivos y propósitos fundantes es una tarea compleja. Alejada del modernismo la educación ambiental no busca principios universales ni dogmas, de otra parte y más cercana a la posmodernidad la educación ambiental se acerca a la diversidad, en todos sus aspectos, social, cultural, económica, natural y epistémica; y da gran relevancia a la historia y al territorio, lo cual hace del objeto de la educación ambiental 
un asunto enmarcado en la complejidad, (Sauve, 1999).

En la construcción conceptual de la educación ambiental se debe empezar por la deconstrucción del concepto de ambiente. En primer lugar desligándolo de la especie de sufijo que le ha impuesto la modernidad al llamarlo "medio ambiente", este rezago tiene orígenes semánticos de la traducción del término inglés "measure", como la designación del espacio físico, un poco lejano del oikos de Platón y Aristóteles, y por otro lado en lo conceptual y epistemológico de la gráfica innumerablemente reproducida para interpretar una seudo integralidad del concepto, donde la interconexión de los círculos (ambiente, economía y cultura), que bien podría ser la figura geométrica del triángulo y que representa toda la visión de la modernidad Kantiana en un solo esquema, la economía por fuera de la sociedad y por fuera de la naturaleza, como dice Marx una supra categoría. Esta categorización es un obstáculo a la propuesta de desarrollo endógeno autogestionado, socio histórico y alternativo.

Entender el concepto de ambiente nos llevará a comprender la educación ambiental como proyecto social, cultural y político. El ambiente es aquel lugar donde confluyen los saberes de la ecología, que integra ciencias naturales y sociales con las relaciones de la conciencia como máxima expresión de la energía, y la cultura. Tal como lo expresa Rivas-Escobar y Luna-Cabrera (2016), "al aceptar la multidimensionalidad del fenómeno humano, aceptamos también que el ambiente es una construcción no solo ecológica sino social, cultural, política y económica".

Estas consideraciones se ven reflejadas de alguna manera en la expresión de uno de nuestro entrevistados:

17:13 I 26, La Educación Ambiental, es un proceso que dura toda la vida y que tiene como objetivo, construir conciencia ambiental, conocimiento ecológico, actividades y valores hacía el medio ambiente, para hacer un compromiso con la Comunidad, sobre el uso racional de todos los recursos que existen en nuestro planeta. in Case 17

En relación con las categorías de currículo y educación ambiental podemos afirmar que una EA con las características señaladas por los docentes participantes espera contribuir a la construcción de un nuevo paradigma, que transforme la educación misma y por consiguiente determine una nueva arquitectura curricular. La estructura curricular de la educación ambiental debe poder articular y entrelazar lo que nunca se debió concebir en forma escindida en el proceso educativo, la formación teórica y la práctica. Desde ese escenario y con la poderosa herramienta del currículo la educación ambiental se puede mover en los campos de la gestión y organización escolar, la investigación educativa y la elaboración de un marco axiológico integrador, complejo y reivindicador de la identidad y el territorio, (Secretaría de educación publica de México, 2012).

Un currículo de estas características debe permitir crear entornos de aprendizaje y vivencias que favorezcan el continuum reflexión-acciónreflexión, y por ese medio la elaboración de la estructura didáctica más pertinente y significativa para los actores del proceso educativo en su contexto sociocultural, privilegiando la identidad y el territorio.

\section{TRANSVERSALIDAD CURRICULAR DE LA DIMENSIÓN AMBIENTAL}

Es muy relevante al interpretar las respuestas de los docentes de las instituciones educativas participantes en la investigación y visualizadas en el diagrama de Sankey de la figura 4, la importancia que le dan a aspectos como la vida, el ambiente, las actitudes y la conciencia, al momento de transversalizar la dimensión ambiental en el currículo. 
La dimensión ambiental transversalizada en el currículo se orienta desde la construcción de valores e incluso conductas donde se redimensiona la relación del ciudadano con sus congéneres y con el entorno natural al que pertenece y que no le pertenece. De esta manera el currículo ambiental debe ser una práctica cotidiana, como lo expresa Florez (2005), de unos valores comunitariamente construidos y ligados al territorio, de carácter social e incluso político, donde el estudiante tenga el escenario propicio para la construcción de una formación ética que le permita entender, analizar, sentir y transformar su ser, su entorno, la sociedad y el mundo.

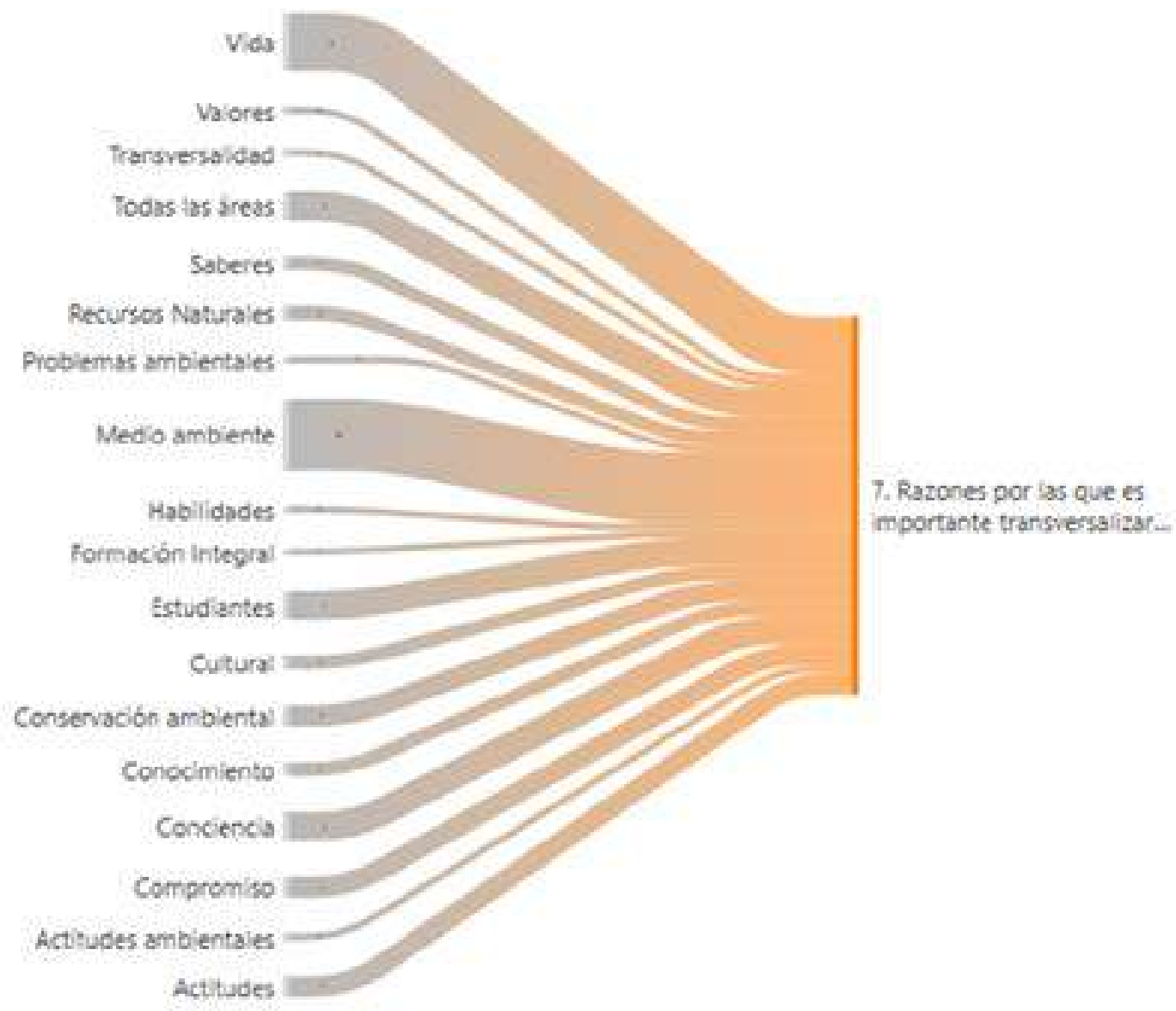

Figura No. 4. Consideraciones de los docentes frente a la importancia de la transversalidad.

Fuente: este estudio

Al analizar las categorías del concepto de transversalidad, la forma como se puede llevar acabo esa transversalización y la importancia de la misma, los docentes de las instituciones educativas de Morasurco y Chachagüí, pudieron establecer relaciones de co-ocurrencia entre elementos que permiten inferir que desde la formulación y desarrollo de los proyectos de herramientas didácticas para la educación ambiental y de transversalidad de la educación ambiental en los currículos escolares, desarrollados por el grupo de investigación PIFIL de la Universidad de Nariño, se ha podido aportar a la construcción de una identidad alternativa desde el punto de vista pedagógico frente a las pociones de la escuela tradicional, ya que como se observa en el diagrama de Sankey de la figura No. 5, las tres categorías mencionadas 
confluyen en aspectos como los saberes, la interdisciplinariedad, la ética ambiental, los saberes y las áreas del conocimiento, los planes de estudio, el proyecto ambiental, la vida y los estudiantes.

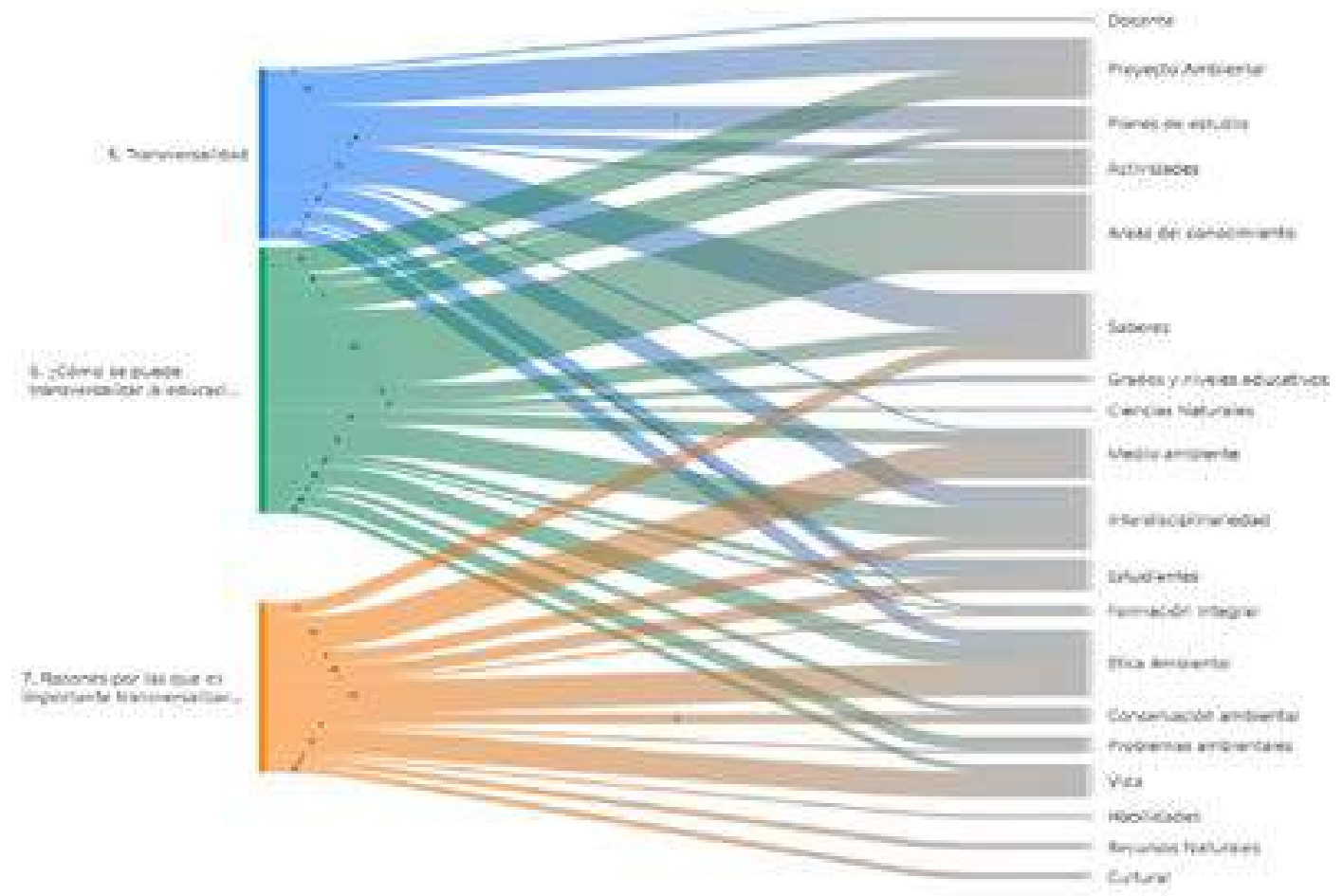

Figura No. 5. Relación entre el concepto de transversalidad, su aplicación e importancia.

Fuente: este estudio

Podemos afirmar con los docentes de las instituciones educativas mencionadas que la transversalidad en el ejercicio diario de los procesos de enseñanza aprendizaje de la educación ambiental es una propuesta para la apertura del pensamiento y una aventura donde se desarrollan fundamentalmente las capacidades para interrelacionar diferentes saberes (que después descubriremos que el conocimiento solo es uno), que vinculen objetos, ideas, fenómenos, conceptos, sentimientos y percepciones que nos permitan entender primero y solucionar después problemas actuales y reales y nos prepare para solucionar los problemas que aún no han surgido, (RodriguezAvila, 2010)
Así considera uno de los participantes de la investigación la importancia de transversalizar la educación ambiental:

15:17 đ 34, Porque la educación ambiental se debe trabajar en equipo, de manera integral y sistémica que abarque los aspectos social, cultural y natural para lograr formar y transformar culturalmente ciudadanos con actitudes que permitan el desarrollo social y sostenible del ambiente. in Case 15

La transversalización de la educación ambiental en particular y de la educación en general implica replantear siglos de historia y praxis educativa y evolucionar hacia una dinámica donde los sujetos del proceso educativo sean ciudadanos aptos para interactuar y responder a problemáticas 
de una realidad cambiante, es decir que desarrollen habilidades incluso, para resolver problemas que aún no se han presentado. La formación de nuevos ciudadanos, debe estar por encima de la transmisión de conocimientos disciplinarios, como menciona Trigos-Carrillo y otros, (2011), debe incluir todas las dimensiones del ser humano en incluir como mínimo algunas de las siguientes habilidades:

- Desarrollo de un pensamiento propio, crítico y reflexivo, con ecuanimidad e independencia de juicio, en nuestro lado del hemisferio en lucha contra el eurocentrismo promovemos, como diría Sabogal (2009), la construcción del pensamiento propio Latinoamericano.

- La comprensión profunda, de raíz, de la realidad circundante y de las relaciones e imbricaciones de las manifestaciones naturales y sociales (Pinilla-Moscoso y Puertas, 2017)

- El trabajo colaborativo y cooperativo, con las habilidades que permitan hacer sinergia de nuestras diferencias y convertir lo que pudiera ser un obstáculo en oportunidades.

- Ética ciudadana, la educación ambiental es fundamentalmente una educación de la ética para el desarrollo de valores que nos permitan entender nuestro lugar en el paraíso, no como los autorizados por la potestad divina para hacer uso indiscriminado y egoísta del jardín del edén, sino como los responsables de garantizar la pervivencia de todas las especies, incluida la nuestra, (Ariza et al, 2017, Vera-Rojas et al, 2017).

- Metacognitivos, aprender a aprender y a desaprender, esto último tal vez lo más difícil. Des cuadricularnos de todo lo que nos han enseñado y que mal hemos aprendido.

- Listos para la acción, como los Scouts, siempre listos, para que una vez sensibilizados ante una realidad problémica o con oportunidades y con el conocimiento para transformar las situaciones hacia escenarios de bien vivir, ejecutemos las acciones y enseñemos a nuestros estudiantes con el ejemplo.

\section{CONCLUSIONES}

Un primer paso para ambientalizar el currículo es el de establecer la relación de los contenidos de las asignaturas con el ambiente. Sin embargo, una tarea anterior, consiste en racionalizar el concepto de ambiente. Algunas iniciativas de ambientalización curricular han optado por relacionar los contenidos disciplinares con los problemas ambientales del entorno institucional y otra vertiente propone relacionar el currículo con el concepto de sostenibilidad.

Las Instituciones Educativas tienen una autonomía parcial al momento de realizar sus construcciones curriculares y sus planes de estudio, debido especialmente a la existencia de los estándares y derechos de aprendizaje que determinan que se debe enseñar, es decir los contenidos de aprendizaje.

El aula no es el único escenario de enseñanza aprendizaje y el diseño curricular para el desarrollo de la educación ambiental debe ser consciente de esta realidad, por lo tanto, debe desde el ejercicio curricular posibilitar los entornos de aprendizaje necesarios para que los estudiantes tengan la posibilidad de demostrar sus fortalezas personales en la escuela incluidas aquellas que no tengan un carácter académico.

Un resultado de la implementación de la educación ambiental debe ser la construcción de empatía, es decir el desarrollo de unos valores 
ciudadanos que nos permitan ponernos en el lugar de los demás. Este es el ejercicio inicial y principal de la transdisciplinariedad, entender que la realidad depende del prisma y perspectiva del observador. El tema pasa por la complejidad que supera el reduccionismo del observador desprevenido que no interactúa con la realidad hasta la dimensión ética de la otredad.

Para poder abordar el tema de la transversalidad en la educación ambiental específicamente en la educación formal, hay que discutir paralelamente el tema del currículo. No se puede transversalizar la educación ambiental sin transformar los rígidos currículos escolares, dependientes de los organismos centrales de educación de cada país en nombre de las determinaciones homogenizantes de los estándares básicos por competencias y del idealizado logro de la calidad medida a través de las pruebas estandarizadas nacionales en internacionales. Los currículos son los primeros elementos por transversalizar, hay que revisar contenidos y métodos, alcances y objetivos.

\section{REFERENCIAS BIBLIOGRÁFICAS}

1. Aldana-Zavala, J., Vallejo-Valdivieso, A., y Isea-Argüelles, J. (2021). Investigación y aprendizaje: Retos en Latinoamérica hacia el 2030. Revista de educación Alteridad. Vol. 16, No. 1, 78-91 https://doi.org/10.17163/alt. v16n1.2021.06

2. Alvira, R. (1986). Dialéctica de la modernidad. Revista anuario filosófico. Universidad de Navarra. Vol (19), No. 2. 9-24. Recuperado: https://hdl.handle.net/10171/2263

3. Angel-Maya, A. (2014). La aventura de los símbolos, una visión ambiental de la historia del pensamiento. Fundación Augusto Angel Maya. Bogotá.

4. Arce-Tovar, D. \& Camacho, S. 2020. Colombia: discursos, prácticas y rupturas de una propuesta en construcción, la educación para el desarrollo y la ciudadanía. Revista Sinergias, Diálogos educativos para la transformación social. Diciembre, 2020. No 11. P 43 - 56.

5. Ariza, C. P., Rueda Toncel, L. Ángel, \& Sardoth Blanchar, J. (2017). La educación ambiental como estrategia global para la sustentabilidad. Revista Boletín Redipe, 6(5), 64-70. Recuperado a partir de https://revista. redipe.org/index.php/1/article/view/258

6. Barrero-Garcia, J. (2019). Concepción de la relación hombre-naturaleza, desde la episteme sujeto-objeto Un acercamiento teórico y experiencial desde la educación ambiental. revista Ideales, p 23. 2019.

7. Flórez O., R. (2005). Pedagogía del Conocimiento. Segunda Edición. Bogotá, Colombia: Ed. Mc Graw Hill.

8. Gutiérrez, J. (1995): Educación ambiental. Fundamentos teóricos, propuestas de transversalidad y orientaciones extracurriculares, Madrid, La Muralla.

9. Gutiérrez, J y Pozo, T. (2006) Modelos teóricos contemporáneos y marcos de fundamentación de la educación ambiental para el desarrollo sostenible. REVISTA IBEROAMERICANA DE EDUCACIÓN. N. ${ }^{\circ} 41$ (2006), pp. 21-68

10. lafrancesco V. Giovanni. Evaluación integral de aprendizajes. Taller. Universidad de Antioquia. Abril 29 y 30 de 2004.

11. Innerarity, D. (1987). Modernidad y posmodernidad. Revista anuario filosófico. Universidad de Navarra. Vol (20), No. 1. 105129. recuperado en https://dadun.unav.edu/ handle/10171/1743

12. Laso-Salvador, S. (2018). La conciencia ambiental de los futuros maestros de educación primaria: diseño de una propuesta didáctica mediante la aplicación de herramientas 
metacognitivas. Tesis doctoral. Universidad de Valladolid. Valladolid, España.

13. Leff, E. (2006). Complejidad, racionalidad ambiental y diálogo de saberes. En: Ceneam. Reflexiones sobre educación ambiental II (pp. 275-284). Segovia, España: Parques Nacionales-Ministerio de Medio Ambiente

14. Ministerio de Educación Nacional. (2016). Derechos básicos de aprendizaje. Ciencias naturales V1. Recuperado de: http:// aprende.colombiaaprende.edu.co/sites/default/ files/naspublic/DBA_C.Naturales.pdf

15. Morín, E. (2000). Los siete saberes necesarios para la educación del futuro. Bogotá: Ministerio de Educación Nacional.

16. Osorio, L. (2015). Aportes a la educación ambiental en Colombia a partir de dos experiencias con instituciones educativas y comunidades indígenas Mhuysqas. Bogotá: Universidad Nacional de Colombia, p. 149.

17. Pinilla Moscoso Cl, Puertas DS. 2017. Cambio climático, gestión y educación ambiental urbana: análisis geopolítico. Estudio de caso: Complejo de humedales en la localidad de 11Suba, Bogotá. bol.redipe [Internet]. 6(11):65-7. Disponible en: https://revista.redipe.org/index. php/1/article/view/401

18. Pita-Morales, L. A. (2016). Línea de tiempo: educación ambiental en Colombia. Praxis, 12(1), 118-125. https://doi. org/10.21676/23897856.1853

19. Rivas-Escobar, H. \& Luna-Cabrera, G. (2016). Ambiente y sostenibilidad, $1^{\mathrm{a}}$. Ed. San Juan de Pasto: Editorial Universitaria, Universidad de Nariño.

20. Rodriguez-Avila, O. 2010. La educación ambiental desde un enfoque interdisciplinar en estudiantes de la carrera ingeniería en agronomía orientada al desarrollo sostenible. Cuadernos de Educación y Desarrollo Vol 2, № 20 (octubre 2010). Recuperado de: http://www. eumed.net/rev/ced/index.htm

21. Sabogal, J. (2009). El desarrollo humano multidimensional. Pasto: Editorial Universitaria, Universidad de Nariño, $230 p$

22. Sauvé, L. (1999). Laeducación ambiental entre la modernidad y la posmodernidad: En busca de un marco de referencia educativo integrador. Tópicos, 1(2). Août 1999, p. 7-27.

23. Secretaría de educación pública de México. 2012. Educación ambiental para la sustentabilidad. Recuperado de https://es.calameo.com/ books/0040256428d013ad54cbb

24. Trigos Carrillo, L.M, Rosales Cueva, J.H., Barboza Fernandes, C.M., Barradas Gerón, M.A., Calderón Villegas, J.J., Enríquez Solano, F., Martínez Rivera, A.A., Quintá Roccato, M.C., Ramos Pismataro, F. y Sabulsky, G.

25. UNESCO. 2012. Educación para el desarrollo sostenible, libro de consulta. Paris.

26. Vera Rojas, M. del P., Chávez Arias, S., \& Torres Lema, M. R. (2017). Educación ambiental: reseña de una experiencia compartida. Revista Boletín Redipe, 6(5), 246-260. Recuperado a partir de https://revista.redipe.org/index.php/1/ article/view/275 